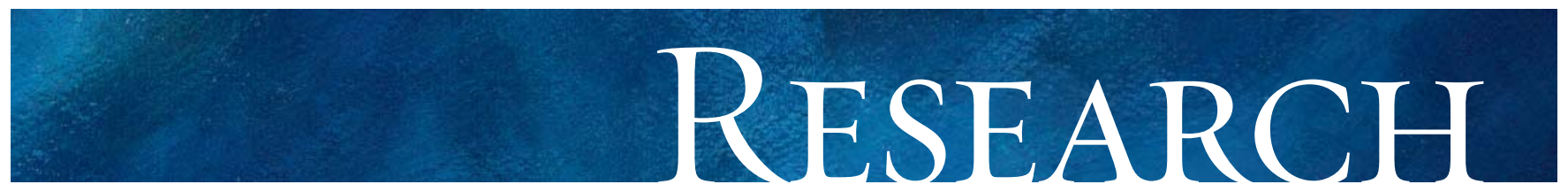

\title{
Predictors of driving cessation in mild-to-moderate dementia
}

\author{
Nathan Herrmann, Mark J. Rapoport, Robert Sambrook, Réjean Hébert, Peter McCracken, \\ Alain Robillard, for the Canadian Outcomes Study in Dementia (COSID) Investigators
}

$\infty$

See related articles, pages 575 and 6oI

\section{ABSTRACT}

Background: Although physicians in most provinces are mandated to report patients whose driving ability is impaired by illness, little is known about dementia-related factors associated with driving cessation. The purpose of our study was to explore factors that may affect the likelihood of driving cessation in a sample of elderly, community-dwelling patients with dementia.

Methods: A 3-year prospective study, the Canadian Outcomes Study in Dementia (COSID) has enrolled 883 patients with mild-to-moderate dementia at 32 centres across Canada. Assessment tools included the Mini-Mental State Examination (MMSE) for cognition, the Global Deterioration Scale (GDS) for staging (severity), the Functional Autonomy Measurement System (SMAF) for function, and the Neuropsychiatric Inventory (NPI) for behaviour. Factors associated with the decision to quit driving after the baseline assessment were tested with Cox survival analysis.

Results: Of 719 subjects who were or had been drivers, 203 $(28.2 \%)$ were still driving at baseline. Over an observation period that averaged 23 months, 97 (48.5\%) of 200 patients quit driving. Factors predictive of driving cessation included GDS (hazard ratio [HR] 1.68, 95\% confidence interval [CI] 1.15-2.45), MMSE score (HR 0.90, 95\% Cl 0.83-0.97) and NPI findings ( $\mathrm{HR} 1.63$ for presence of $\geq 3$ behaviours, $95 \% \mathrm{Cl}$ 1.01-2.62). Among the NPI behaviours, when they were analyzed separately, agitation led to a decreased likelihood of driving cessation $(p=0.019)$, whereas apathy $(p=0.031)$ and hallucinations $(p=0.050)$ led to an increased likelihood.

Interpretation: Cognitive impairment and behaviours such as agitation, apathy and hallucinations were significant predictors of driving cessation in patients with a mild to moderate degree of dementia. These findings should be considered when one counsels patients and their families.

CMAJ 2006;175(6):591-5

$\Lambda$ landmark study of the epidemiology of dementia, the Canadian Study on Health and Aging (CSHA) determined that in I992, some 3I6 500 Canadians over the age of 65 years ( 1 in ${ }_{3} 3$ ) had dementia, with the risk of the disease doubling for every 5 years of age after $65 \cdot{ }^{1,2}$ With the in- creasing mean age of our population, it is estimated that 500000 Canadians will have dementia by 202I, and 750000 by 203I. ${ }^{3}$ Although incidents involving drivers affected by dementia frequently make the headlines in the lay press, there is surprisingly little data on the factors in dementia that may be associated with driving cessation. In a cross-sectional study of community-dwelling subjects, Foley and colleagues ${ }^{4}$ found that $46 \%$ of those whose dementia was rated as questionable (i.e., with a Clinical Dementia Rating ${ }^{5}$ [CDR] of 0.5) and $22 \%$, as mild (CDR I) were still driving. Indeed, Duchek and associates ${ }^{6}$ found that of patients who passed an on-road test at baseline, only about $20 \%$ of those with questionable dementia and none of those with mild dementia passed another road test 2 years later. In a retrospective study of 430 patients at a memory clinic, age, an urban dwellingplace, level of cognitive function and relative ability to perform the activities of daily living were found to be predictive of driving cessation. ${ }^{7}$ Similarly, in a small prospective study of 53 patients with dementia, age and Mini-Mental State Examination $(\mathrm{MMSE})^{8}$ score predicted driving cessation over the next 2-3 years. ${ }^{9}$ Determination of the factors associated with driving cessation is important, given the well-documented association between the risk of being involved in a motorvehicle collision and the severity and duration of the dementia. ${ }^{10}$

Clinical practice guidelines have not been consistently helpful in providing physicians with guidance related to driving and dementia. For example, although the conclusions of the Canadian Consensus Conference on Dementia ${ }^{3}$ emphasized the need to assess patients with dementia for fitness to drive and provided suggestions for factors requiring evaluation (e.g., cognition, function, medical status, medication review), there did not provide specific guidelines for how to determine when driving cessation was necessary. In contrast, the American Academy of Neurology practice parameter for risk of driving in cases of Alzheimer's disease ${ }^{11}$ provides much clearer recommendations for driving cessation, based on CDRs. Unfortunately, most primary care physicians are unfamiliar with CDRs and may have difficulty using athe ratings in everyday practice. The guide published by the Canadian Medical Association ${ }^{12}$ quotes the National Safety Code, which has suggested that patients whose MMSE score is less than 24 are ineligible to hold a driver's license unless they have undergone a complete neurologic assessment. Unfortu- 


\section{Box 1: Criteria used for diagnoses of dementia, by type}

AD Criteria of the National Institute of Neurological and Communicative Diseases

Alzheimer's Disease and Related Disorders Association criteria (Neurology 1984;34:939)

VaD National Institute of Neurological Disorders and Stroke L'Association Internationale pour la Recherche et l'Enseignement en Neurosciences (Neurology 1993;43:250)

FTD Lund-Manchester criteria (J Neurol Neurosurg Psychiatry 1994;57:416)

DLB Consensus guidelines of the Consortium on Dementia with Lewy Bodies (Neurology 1996;47:1113)

Note: $A D=$ Alzheimer's disease, $\mathrm{VaD}=$ vascular dementia, $\mathrm{FTD}=$ frontotemporal dementia, DLB = dementia with Lewy bodies.

nately, what constitutes a neurologic assessment is left unspecified. How a score of 24 on the MMSE was chosen and why a "neurological" as opposed to a geriatric medical or psychiatric assessment is required are not explained. Although several other practice guidelines have addressed the issue, ${ }^{13-15}$ physicians still require more guidance.

The purpose of our study was to explore the factors affecting the likelihood of driving cessation in a sample of elderly, community-dwelling people with dementia. Knowledge of these factors could help physicians identify patients at risk, in order to help them begin to plan for alternatives to driving.

\section{Methods}

The Canadian Outcomes Study in Dementia (COSID) used a modified, nonprobabilistic cluster sampling method to conduct a 3-year prospective pharmacoepidemiological study at 32 academic and community sites across Canada. A detailed description of the methodology and baseline patient characteristics has been published previously. ${ }^{16}$ Briefly, the study centres were situated in 5 major regions of Canada (British Columbia, the Prairies, Ontario, Québec and the Atlantic Provinces) with investigators representing the specialties of geriatric medicine $(n=\mathrm{I} 6)$, geriatric psychiatry $(n=7)$ and neurology $(n=6)$ as well as family practice $(n=3)$. The study was approved by the site's local review ethics board or, where such boards were unavailable, by a central ethics board; written informed consent was obtained from all patients as well as from their caregivers. Inclusion criteria included an age of 60 years or older, dwelling in the community (as opposed to a seniors' residence or long-term care facility), a DSM-IV ${ }^{17}$ diagnosis of dementia (Box I) and a Global Deterioration Score ${ }^{18}$ [GDS] of 5 or less (indicating early or mild disease severity).

Information and assessments were collected at baseline and every 6 months for 3 years or until death, entry into an institution, study withdrawal or loss to follow-up. Assessment included measures of cognition (the Modified MiniMental State Examination [3MS]), ${ }^{19}$ global staging or severity (GDS) ${ }^{18}$ function (Functional Autonomy Measurement System $[\mathrm{SMAF}])^{20}$ and behaviour (the Neuropsychiatric Inventory $[\mathrm{NPI}]) .{ }^{21}$ The $3 \mathrm{MS}$ is used to measure orientation,

Table 1: Baseline characteristics of study participants, by driving status

No. (\%), unless otherwise indicated

\begin{tabular}{|c|c|c|c|c|}
\hline Demographic or other characteristic & $\begin{array}{l}\text { Still driving } \\
\quad n=203\end{array}$ & $\begin{array}{c}\text { No longer driving } \\
n=516\end{array}$ & $\begin{array}{c}\text { Never drove } \\
n=164\end{array}$ & $\begin{array}{l}\text { Overall } \\
n=883\end{array}$ \\
\hline Age, mean (standard deviation), yr & $74.55(6.37)$ & $77.19(6.23)$ & $79.24(5.90)$ & $76.96 \quad(6.38)$ \\
\hline Female & $55(11.5)$ & $276(57.7)$ & $147(30.8)$ & 478 \\
\hline Male & $148(36.5)$ & $240(59.3)$ & $17 \quad(4.2)$ & 405 \\
\hline Alzheimer's disease & $175(24.0)$ & $413(56.7)$ & $140(19.2)$ & 728 \\
\hline Other dementia & $28(18.1)$ & $103(66.5)$ & $24(15.5)$ & 155 \\
\hline Single, including widowed, separated or divorced & $28 \quad(9.9)$ & $159(56.0)$ & $97(34.2)$ & 284 \\
\hline Married or common-law & $175(29.2)$ & $357(59.6)$ & $67(11.2)$ & 599 \\
\hline \multicolumn{5}{|l|}{ Region of residence } \\
\hline Atlantic provinces & $17(13.6)$ & $79(63.2)$ & $29(23.2)$ & 125 \\
\hline British Columbia & $17(18.1)$ & $58(61.7)$ & $19(20.2)$ & 94 \\
\hline Ontario & 95 (21.9) & $270(62.2)$ & $69(15.9)$ & 434 \\
\hline Prairie provinces & $34(41.5)$ & $37(45.1)$ & $11(13.4)$ & 82 \\
\hline Quebec & $40(27.0)$ & $72(48.6)$ & $36(24.3)$ & 148 \\
\hline Residence in a province with mandatory reporting & $152(21.7)$ & $427(61.0)$ & $121(17.3)$ & 700 \\
\hline \multicolumn{5}{|l|}{ Test results, mean (standard deviation) } \\
\hline Neuropsychiatric Inventory & $8.79(11.66)$ & $12.26(12.77)$ & $12.41(14.43)$ & $11.49(12.92)$ \\
\hline Functional Autonomy Measurement System (SMAF) & $11.61(7.33)$ & $20.79 \quad(9.40)$ & $19.09(10.84)$ & $18.40 \quad(9.98)$ \\
\hline Mini-Mental State Examination & $24.25(3.33)$ & $21.33 \quad(4.94)$ & $20.59 \quad(5.45)$ & $21.87 \quad(4.91)$ \\
\hline
\end{tabular}


registration attention, calculation, recall, language, praxis and executive function. Because the $3 \mathrm{MS}$ tests a broader range of cognitive functions than the MMSE, MMSE scores can be derived from the $3 \mathrm{MS}$. The SMAF is a well tested and validated 29 -item scale that measures functional ability in 5 dimensions: basic and instrumental activities of daily living, mobility, communication and mental function. The NPI, a I2-item scale, assesses the frequency and severity of delusions, hallucinations, agitation or aggression, depression or dysphoria, anxiety, euphoria or elation, apathy or indifference, disinhibition, irritability or lability, aberrant motor behaviours, and sleep and appetite disturbances. Data collected at each site were analyzed centrally by Syreon Corporation (Vancouver, BC).

Factors associated with the later decision to quit driving (i.e., after the baseline interview) were examined with an exploratory Cox survival analysis. The model included gender, age, marital status, dementia type (Alzheimer's v. other), income, education level (completed grade school v. high school and above), geographic region, GDS, MMSE, SMAF, NPI (presence of $\geq 3$ behaviours v. $<3$ behaviours), number of medical comorbidities, total number of medications, number of psychoactive medications and time since initiation of treatment with a cholinesterase inhibitor. In addition, the model included the variable of provincial mandatory reporting requirements. At the time of this report, Québec, Nova Scotia and Alberta had discretionary reporting, whereas all other provinces had mandatory reporting. An additional Cox regression analysis was conducted to examine the contribution of each of the I2 NPI domains. To reduce the risk of overfitting the data, the number of predictor variables in this model was reduced by means of backward elimination. Dementia type, age and gender, however, were retained in the model to ensure that the results were adjusted for these important variables.

\section{Results}

Our sample consisted of 883 patients with an average age of 77.0 years (standard deviation [SD] 6.4), and a mean MMSE score of 2I.9 (SD 4.9); 728 (82.4\%) had Alzheimer's disease. Baseline characteristics are listed in Table I by driving status. Of the total, 719 people (8I.4\%) were still or had been drivers; at baseline, $203(28.2 \%)$ of them were still actively driving. Among the 516 people who had already ceased driving at the time of the baseline interview, the reported reasons for cessation included involvment in a motor vehicle collision $(n=24$, $4.7 \%$ ), vision problems ( $n=24,4.7 \%$ ), having their licence formally revoked ( $n=60$, II. $6 \%$ ) or letting it expire $(n=6$, I. $2 \%)$.

A Cox survival analysis examined the factors associated with driving cessation in those who were active drivers at baseline over an average follow-up period of 23 months. The proportion of active drivers remaining throughout the observation period is shown as a "survival" curve in Fig. I. Of the 203 active drivers at baseline, 148 (72.9\%) were men. Data for analysis were available for 200 drivers, of whom 97 (48.5\%) stopped driving during the observation period.

The factors that significantly increased the probability of quitting driving included GDS stage $(p<0.008), \operatorname{MMSE}(p=$ 0.009 ) and the presence of 3 or more behavioural disturbances $(p=0.047)$. Hazard ratios for all variables are reported in Table 2. Backward elimination of the least important variables from the model confirmed that higher GDS stage ( $p$ $=0.0 \mathrm{I}$ ), lower MMSE (i.e., greater cognitive impairment; $p$ $=0.009$ ) and NPI ( $\geq 3$ behaviours; $p=0.024$ ) were significant predictors of driving cessation; education level (lack of high school) also became significant in this model $(p=0.045)$. Introduction of the I 2 individual NPI behaviours into the model revealed agitation/aggression ( $p=0.019)$, apathy $(p=0.03 \mathrm{I})$ and hallucinations $(p=0.050)$ as significant behaviours. The presence of apathy or hallucinations were predictive of driving cessation, whereas the presence of agitation/aggression was associated with a reduced risk of stopping driving (Table 3). Reported reasons for driving cessation after baseline included having been involved in a motor vehicle collision ( $n$ $=4,4.1 \%$ of 97$)$, vision problems $(n=2,2.1 \%)$, formal revocation of the driver's licence $(n=\mathrm{I} 8, \mathrm{I} 8.6 \%)$ and nonrenewal after licence expiration $(n=3,3.1 \%)$.

\section{Interpretation}

In this sample of patients with a diagnosis of dementia, $28 \%$ were still driving at the baseline assessment. Increased cognitive and global impairment and the presence of a greater number of behavioural disturbances all increased the likelihood of driving cessation during the next few years. In our analysis gender was not a significant factor, possibly because the sample was skewed: $73 \%$ of the active drivers were men. Similarly, the length of time a patient had been treated with a cholinesterase inhibitor was not significant, possibly because at baseline most patients were being so treated. Finally, living in a province with mandatory reporting had no significant effect on increasing the risk of driving cessation. COSID findings confirmed the associations previously documented of driving cessation with disease severity, increasing cognitive impairment and age. ${ }^{4-7,9}$ These same factors have also been

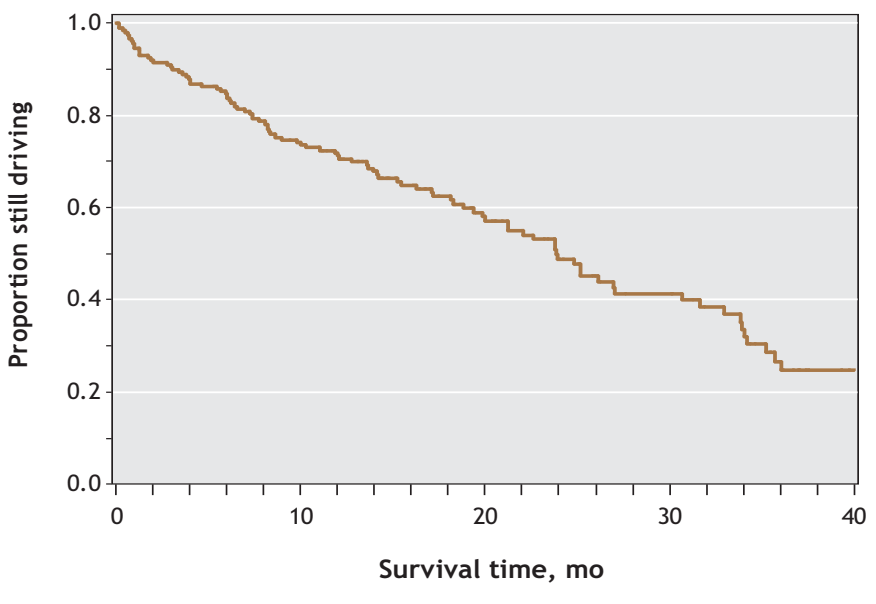

Fig 1: "Survival" curve of the 203 active drivers (i.e., a trace showing the proportion who continued driving) over the study period. 
shown to increase the risk of motor-vehicle collisions in people with dementia. ${ }^{10}$ Male gender has been associated with a higher risk of crashes and moving violations; ${ }^{22}$ it is notable that at baseline, we found women to be significantly likelier than men to have already quit driving.

In this study, having 3 or more behavioural disturbances increased the risk of driving cessation by more than $60 \%$. The relation between behavioural disturbances and driving in dementia has not previously been examined. We speculate that hallucinations, which increased the likelihood of driving cessation more than 2 -fold, may have acted as a marker of disease severity that alerted physicians and families to the need for a patient to quit driving. Apathy, which increased the likelihood of driving cessation by $70 \%$, may have led drivers to quit on their own. Finally, agitation/aggression reduced the risk of driving cessation, possibly because families and physicians might be more uncomfortable confronting such people with the need to stop driving.

The limitations of this study included the generalizability of the results. Although COSID sampled a large number of elderly individuals with dementia across Canada from both community and academic sites, the included patients were managed mostly by specialists; it is therefore unclear how representative our sample might be of the general population of patients who have dementia. The baseline characteristics

Table 2: Hazard ratios for driving cessation, by characteristic

\begin{tabular}{|l|l|}
\hline Characteristic or variable & $\mathrm{HR}(95 \% \mathrm{Cl})$ \\
\hline Alzheimer's type of dementia & $0.62(0.32-1.18)$ \\
\hline Age* & $1.03(1.00-1.07)$ \\
\hline Female sex & $1.49(0.88-2.53)$ \\
\hline Education beyond grade school & $1.46(0.85-2.53)$ \\
\hline Married or living common-law & $0.82(0.42-1.60)$ \\
\hline Region of residence & \\
\hline British Columbia & $0.61(0.18-2.08)$ \\
\hline Ontario & $0.98(0.45-2.13)$ \\
\hline Prairie provinces & $0.94(0.40-2.20)$ \\
\hline Quebec & $1.12(0.35-3.57)$ \\
\hline Residence where reporting is mandatory & $1.41(0.53-3.74)$ \\
\hline Household income* & $1.05(0.93-1.19)$ \\
\hline Time from initiation of cholinesterase inhibitor & $1.17(0.86-1.59)$ \\
\hline Comorbidities, no. & $0.95(0.88-1.03)$ \\
\hline Concomitant medications, no. & $1.00(0.87-1.14)$ \\
\hline Psychoactive medications, no. & $1.17(0.80-1.70)$ \\
\hline Neuropsychiatric test results† & \\
\hline Global Deterioration Scale* & $1.68(1.15-2.45)$ \\
\hline Mini-Mental State Examination: MMSE* & $0.90(0.83-0.97)$ \\
\hline Functional autonomy: SMAF & $1.00(0.97-1.04)$ \\
\hline Neuropsychiatric Inventory, $\geq 3$ behaviours & $1.63(1.01-2.62)$ \\
\hline Ne: & \\
\hline
\end{tabular}

Note: $\mathrm{HR}=$ hazard ratio, $\mathrm{Cl}=$ confidence interval, $\mathrm{SMAF}=$ Functional Autonomy Measurement System.

*Continuous variables, which underwent continuous analysis.

tExcept for MMSE, higher scores indicate worse condition. of participants in COSID are similar, however, to those of the epidemiological population sampled by CSHA. ${ }^{1}$ Another limitation was the inability to identify the presence or absence of other drivers associated with our subjects, a factor that would also affect driving cessation. Finally, it is unclear whether the predictors identified in this study led to physicians recommending driving cessation or reporting these patients to provincial transport ministries, to family members demanding that their relatives quit or to patients deciding on their own to stop driving.

Should we be concerned that $28 \%$ of older people with cognitive and functional impairment severe enough to warrant a diagnosis of dementia were driving at baseline? Is it worrisome that only $12 \%-19 \%$ of these patients had their licences formally revoked, and that provincial legislation requiring mandatory reporting had so little apparent effect on driving cessation? These questions are difficult to answer. Although many studies have shown an increased risk of motor vehicle collisions among people with dementia, some patients with mild dementia nevertheless may be able to drive safely. Unfortunately, this study does not provide information on how to assess the safety risk, nor is there much agreement about which methods (e.g., neuropsychological testing, driving simulators, on-road testing, etc.) would best predict collision risk..$^{10}$ On the other hand, the fact that cognitive impair-

Table 3: Association of neurospsychiatric behaviours and other factors at baseline with later driving cessation*

\begin{tabular}{|lc|}
\hline Characteristic or variable & $\mathrm{HR}(95 \% \mathrm{Cl})$ \\
\hline Alzheimer's type of dementia & $0.73(0.39-1.39)$ \\
\hline Age & $1.03(0.99-1.07)$ \\
\hline Female sex & $1.53(0.97-2.42)$ \\
\hline Education beyond grade school & $1.38(0.82-2.32)$ \\
\hline Neuropsychiatric test scores & \\
\hline Global Deterioration Scale stage & $1.67(1.15-2.44)$ \\
\hline Mini-Mental State Examination score & $0.90(0.83-0.97)$ \\
\hline Behaviour-related risk factors & \\
\hline Delusions & $1.12(0.52-2.40)$ \\
\hline Hallucinations & $2.57(1.00-6.60)$ \\
\hline Agitation /aggression & $0.54(0.32-0.90)$ \\
\hline Depression/dysphoria & $1.01(0.63-1.65)$ \\
\hline Anxiety & $1.29(0.78-2.15)$ \\
\hline Euphoria/elation & $1.09(0.45-2.63)$ \\
\hline Apathy/indifference & $1.69(1.05-2.72)$ \\
\hline Disinhibition & $1.00(0.45-2.19)$ \\
\hline Irritability/lability & $1.03(0.61-1.75)$ \\
\hline Aberrant motor behaviour & $1.01(0.52-1.96)$ \\
\hline Nighttime behaviour & $1.02(0.53-1.96)$ \\
\hline Appetite/eating disorder & $0.94(0.53-1.66)$ \\
\hline
\end{tabular}

Note: $\mathrm{HR}=$ hazard ratio, $\mathrm{Cl}=$ confidence interval.

*Cox model with individual Neuropsychiatric Inventory (NPI) behaviours, after adjusting for dementia type, age, gender and the most important clinical factors remaining after backward elimination.

†Lower scores indicate worse cognition. 
ment and behavioural disturbances - both easily measurable in the clinician's office - were strong predictors of driving cessation suggests that measures already in use by clinicians to monitor patients may also serve to warn of increases in the risks of driving as patients' abilities decline.

This article has been peer reviewed.

From Sunnybrook Health Sciences Centre and the Department of Psychiatry, University of Toronto (Herrmann, Rapoport), Toronto, Ont.; Syreon Corporation (Sambrook), Vancouver, BC; Sherbrooke University (Hébert), Sherbrooke, and the University of Montreal (Robillard), Montréal, Que.; and Glenrose Rehabilitation Hospital (McCracken), Edmonton, Alta.

Competing interests: Funding for COSID (the Canadian Outcomes Study in Dementia) was provided by an unrestricted grant from Janssen Ortho Inc.

Contributors: All authors participated in the conception and design of the study and the analysis and interpretation of the data. Herrmann, Hébert, McCracken and Robillard acquired the data; Herrmann, Rapaport and Sambrook drafted the manuscript. All authors revised the article for content and approved the version to be published.

\section{REFERENCES}

I. CSHA Working Group. Canadian Study on Health and Aging: study methods and prevalence of dementia. $C M A J$ I994;150(6):899-913.

2. The Canadian Study of Health and Aging Working Group. The incidence of dementia in Canada. Neurology 2000;55:66-73.

3. Patterson CJS, Gauthier S, Bergman $\mathrm{H}$, et al. The recognition, assessment and management of dementing disorders: conclusions from the Canadian Consensus Conference on Dementia. CMAJ 1999;I6o(I2 Suppl):Si-S15. Available: www.cmaj .ca/cgi/data/I6o/I2/DCI/I (accessed 2006 Jul Ig).

4. Foley DJ, Masaki KH, Ross GW, et al. Driving cessation in older men with incident dementia. J Am Geriatr Soc 2000;48:928-30.

5. Morris JC. The Clinical Dementia Rating (CDR): current version and scoring rules. Neurology 1993;43:2412-4.

6. Duchek JM, Carr DB, Hunt L, et al. Longitudinal driving performance in earlystage dementia of the Alzheimer type. J Am Geriatr Soc 2003;51:1342-7.

7. Talbot A, Bruce I, Cunningham CJ, et al. Driving cessation in patients attending a memory clinic. Age Ageing 2005;34:363-8.

8. Folstein MF, Folstein SE, McHugh PR. "Mini-Mental State": a practical method for grading the cognitive state of patients for the clinician. J Psychiatr Res 1975;12:189-98.

9. Adler G, Kuskowski M. Driving cessation in older men with dementia. Alzheimer Dis Assoc Disord 2003; 17:68-7I.

Io. Brown LB, Ott BR. Driving and dementia: a review of the literature. J Geriatr Psychiatry Neurol 2004;17:232-40.

II. Dubinsky RM, Stein AC, Lyons K. Practice parameter: risk of driving and Alzheimer's disease (an evidence-based review). Neurology 2000;54:2205-II.

I2. Canadian Medical Association. Determining medical fitness to drive: a guide for physicians. 6th ed. Ottawa: Canadian Medical Association; 2000. p. 56-7. Available: www.aoneuro.on.ca/FitnesstoDrive/contents.pdf (accessed 2006 Jul I9).

I3. Johansson K, Lundberg C. The Ig94 International Consensus Conference on Dementia and Driving: a brief report. Alzheimer Dis Assoc Disord I997;II:62-9.
14. American Psychiatric Association. Practice guideline for the treatment of patients with Alzheimer's disease and other dementias of late life. Am J Psychiatry 1997; I54 (5 Suppl):I-39.

I5. Small GW, Rabins PV, Barry PP, et al. Diagnosis and treatment of Alzheimer disease and related disorders. JAMA I997; 278:1363-71.

I6. Sambrook R, Herrmann N, Hébert R, et al. Canadian Outcomes Study in Dementia: study methods and patient characteristics. Can J Psychiatry 2004;49:417-27.

17. American Psychiatric Association. Diagnostic and statistical manual of mental disorders: DSM-IV. 4th ed. Washington (DC): The Association; I994.

I8. Reisberg B, Ferris SH, de Leon MJ, et al. The Global Deterioration Scale for assessment of primary degenerative dementia. Am J Psychiatry 1982;139:1136-9.

I9. Teng EL, Chiu HC. The Modified Mini-Mental State (3MS) examination. J Clin Psychiatry I987;48:314-8.

20. Hébert R, Carrier R, Bilodeau A. The Functional Autonomy Measurement System (SMAF): description and validation of an instrument for the measurement of handicaps. Age Ageing 1988;17:293-302.

2I. Cummings JL, Mega M, Gray K, et al. The Neuropsychiatric Inventory: comprehensive assessment of psychopathology in dementia. Neurology 1994;44:2308-I4.

22. Massie DL, Green PE, Campbell KL. Crash involvement rates by driver gender and the role of average annual mileage. Accid Anal Prev 1997;29:675-85.

Correspondence to: Dr. Nathan Herrmann, Department of Psychiatry, Sunnybrook Health Sciences Centre, 2075 Bayview Avenue, Toronto ON M4N 3M5; tel $4{ }_{1} 6$ 480-6133;

fax 4I6 480-6022; n.herrmann@utoronto.ca

\section{Editor's take}

- Cognitively impaired patients who drive are a worry to practitioners in all specialties. The decision about for whom and when to forbid driving is a difficult matter of judgment because little is known, not only generally about the actual risks, but also particularly about individual limitations.

- This study found that most of the patients who had been identified with some cognitive impairment had already quit driving. Factors that were associated with future quitting were degree of cognitive impairment and some neurobehavioural features.

Implications for practice: We need to know more about the decision to quit and about the effects of other factors such as the need to drive and the availability of a substitute driver. Moreover, the collision record of cognitively impaired drivers, and its predictors, is a robust outcome that deserves further scrutiny.

\section{ACCESS}

$C M A J$ is the only leading general medical journal that is free online. CMAJ.ca receives over 2 million hits per month of which two-thirds are from international readers. 